Bull. Korean Math. Soc. 52 (2015), No. 2, pp. 571-579

http://dx.doi.org/10.4134/BKMS.2015.52.2.571

\title{
CENTROID OF TRIANGLES ASSOCIATED WITH A CURVE
}

\author{
Dong-Soo Kim And Dong Seo Kim
}

\begin{abstract}
Archimedes showed that the area between a parabola and any chord $A B$ on the parabola is four thirds of the area of triangle $\triangle A B P$ where $P$ is the point on the parabola at which the tangent is parallel to the chord $A B$. Recently, this property of parabolas was proved to be a characteristic property of parabolas. With the aid of this characterization of parabolas, using centroid of triangles associated with a curve we present two conditions which are necessary and sufficient for a strictly locally convex curve in the plane to be a parabola.
\end{abstract}

\section{Introduction}

We study strictly locally convex plane curves. Recall that a regular plane curve $X: I \rightarrow \mathbb{R}^{2}$ in the plane $\mathbb{R}^{2}$, where $I$ is an open interval, is called convex if, for all $s \in I$ the trace $X(I)$ of $X$ lies entirely on one side of the closed half-plane determined by the tangent line at $X(s)([4])$. A regular plane curve $X: I \rightarrow \mathbb{R}^{2}$ is called locally convex if, for each $s \in I$ there exists an open subinterval $I_{0} \subset I$ containing $s$ such that the curve $\left.X\right|_{I_{0}}$ restricted to $I_{0}$ is a convex curve.

Henceforth, we will say that a locally convex curve $X$ in the plane $\mathbb{R}^{2}$ is strictly locally convex if the curve is smooth (that is, of class $C^{(3)}$ ) and is of positive curvature $\kappa$ with respect to the unit normal $N$ pointing to the convex side. Hence, in this case we have $\kappa(s)=\left\langle X^{\prime \prime}(s), N(X(s))\right\rangle>0$, where $X(s)$ is an arc-length parametrization of $X$.

When $f: I \rightarrow \mathbb{R}$ is a smooth function defined on an open interval $I$, we will also say that $f$ is strictly convex if the graph of $f$ has positive curvature $\kappa$ with respect to the upward unit normal $N$. This condition is equivalent to the positivity of $f^{\prime \prime}(x)$ on $I$.

Received March 14, 2014; Revised May 30, 2014.

2010 Mathematics Subject Classification. 53A04.

Key words and phrases. centroid, parabola, triangle, plane curvature, strictly locally convex curve.

The first author was supported by Basic Science Research Program through the National Research Foundation of Korea (NRF) funded by the Ministry of Education, Science and Technology (2010-0022926). 
Suppose that $X$ is a strictly locally convex curve in the plane $\mathbb{R}^{2}$ with the unit normal $N$ pointing to the convex side. For a fixed point $P \in X$, and for a sufficiently small $h>0$, we consider the line $\ell$ passing through $P+h N(P)$ which is parallel to the tangent line $t$ of $X$ at $P$ and the points $A$ and $B$ where the line $\ell$ intersects the curve $X$. We denote by $t_{1}, t_{2}$ the tangent lines of $X$ at the points $A, B$ and by $Q, A_{1}, B_{1}$ the intersection points $t_{1} \cap t_{2}, t_{1} \cap t, t_{2} \cap t$, respectively.

We let $L_{P}(h), g_{P}(h), j_{P}(h)$ and $k_{P}(h)$ denote the length of the chord $A B$, the distance from the centroid $G$ of the section of $X$ cut off by $\ell$ to the line $\ell$, the distance from the centroid $J$ of the triangle $\triangle Q A B$ to the line $\ell$ and the distance from the centroid $K$ of the triangle $\triangle Q A_{1} B_{1}$ to the line $\ell$, respectively.

Now, we consider $S_{P}(h)$ and $T_{P}(h)$ defined by the area of the region bounded by the curve $X$ and chord $A B$, the area $|\triangle P A B|$ of triangle $\triangle P A B$, respectively. Then, obviously we have

$$
T_{P}(h)=\frac{1}{2} h L_{P}(h)
$$

and we get $([9])$

$$
\frac{d}{d h} S_{P}(h)=L_{P}(h)
$$

It is well known that parabolas satisfy the following properties.

Proposition 1. Suppose that $X$ is an open part of a parabola. Then we have the following.

1) For arbitrary point $P \in X$ and sufficiently small $h>0, X$ satisfies

$$
S_{P}(h)=\frac{4}{3} T_{P}(h) .
$$

2) For arbitrary point $P \in X$ and sufficiently small $h>0, X$ satisfies

$$
g_{P}(h)=\frac{2}{5} h .
$$

3) For arbitrary point $P \in X$ and sufficiently small $h>0, X$ satisfies

$$
j_{P}(h)=\frac{2}{3} h .
$$

4) For arbitrary point $P \in X$ and sufficiently small $h>0, X$ satisfies

$$
k_{P}(h)=\frac{4}{3} h .
$$

Proof. For a proof of 1), see [16]. If we denote by $V$ the point where the parallel line $m$ through the point $P$ to the axis of $X$ meets the chord $A B$, then $V$ is the mid point of $A B$ and the point $Q$ is on the line $m$ with $P V=P Q$. This completes the proof of 2), 3) and 4). 
Very recently, the first author of the present paper and Y. H. Kim showed that among strictly convex plane curves, the above area property (1.3) of parabolic sections characterize parabolas. More precisely, they proved as follows $([9])$.

Proposition 2. Let $X$ be a strictly convex curve in the plane $\mathbb{R}^{2}$. Then $X$ is a parabola if and only if it satisfies

(C) For a point $P$ on $X$ and a chord $A B$ of $X$ parallel to the tangent of $X$ at $P$, the area of the region bounded by the curve and $A B$ is $4 / 3$ times the area of triangle $\triangle A B P$, that is,

$$
S_{P}(h)=\frac{4}{3} T_{P}(h)
$$

Archimedes showed that parabolas satisfy (1.3) ([16]). Actually, in [9] the first author of the present paper with Y. H. Kim established five characterizations of parabolas, which are the converses of well-known properties of parabolas originally due to Archimedes ([16]). For some properties and characterizations of parabolas with respect to the area of triangles associated with a curve, see $[3,10,13,14]$. For the higher dimensional analogues of some results in [9], see [7] and [8].

In [11], using Proposition 1, D.-S. Kim et al. proved the following characterization theorem for parabolas with respect to the function $g_{P}(h)$.

Proposition 3. Let $X$ be a strictly locally convex plane curve in the plane $\mathbb{R}^{2}$. For a fixed point $P$ on $X$ and a sufficiently small $h>0$, we denote by $\ell$ the parallel line through $P+h N(P)$ to the tangent $t$ of the curve $X$ at $P$. We let $g_{P}(h)$ the distance from the center $G$ of gravity of the section of $X$ cut off by $\ell$ to the line $\ell$. Then $X$ is an open part of a parabola if and only if it satisfies for a fixed point $P$ on $X$ and a sufficiently small $h>0$

$$
g_{P}(h)=\frac{2}{5} h
$$

In [11], the distance from the center $G$ of gravity of the section of $X$ cut off by $\ell$ to the tangent $t$ of $X$ at $P$ was denoted by $d_{P}(h)$. Hence, we see that $d_{P}(h)+g_{P}(h)=h$.

In this article, we study whether the remaining properties of parabolas in Proposition 1 characterize parabolas.

First of all, in Section 2 we prove the following:

Theorem 4. Suppose that $X$ denotes a strictly locally convex plane curve in the plane $\mathbb{R}^{2}$. For a fixed point $P$ on $X$ and a sufficiently small $h>0$, we denote by $\ell$ the parallel line through $P+h N(P)$ to the tangent $t$ of the curve $X$ at $P$. Then we have

$$
\lim _{h \rightarrow 0} \frac{j_{P}(h)}{h}=\frac{2}{3}
$$


and

$$
\lim _{h \rightarrow 0} \frac{k_{P}(h)}{h}=\frac{4}{3} .
$$

Finally, with the aid of the characterization theorem of parabolas (Theorem 3 in [9]), in Section 4 we prove the following.

Theorem 5. Suppose that $X$ denotes a strictly locally convex $C^{(3)}$ curve in the plane $\mathbb{R}^{2}$. Then the following are equivalent.

1) For all $P \in X$ and sufficiently small $h>0, X$ satisfies

$$
j_{P}(h)=\lambda(P) h^{\mu(P)},
$$

where $\lambda(P)$ and $\mu(P)$ are some functions.

2) For all $P \in X$ and sufficiently small $h>0, X$ satisfies

$$
j_{P}(h)=\frac{2}{3} h .
$$

3) $X$ is an open part of a parabola.

For the function $k_{P}(h)$, the similar argument as in the proof of Theorem 5 yields the following.

Theorem 6. Suppose that $X$ denotes a strictly locally convex $C^{(3)}$ curve in the plane $\mathbb{R}^{2}$. Then the following are equivalent.

1) For all $P \in X$ and sufficiently small $h>0, X$ satisfies

$$
k_{P}(h)=\lambda(P) h^{\mu(P)},
$$

where $\lambda(P)$ and $\mu(P)$ are some functions.

2) For all $P \in X$ and sufficiently small $h>0, X$ satisfies

$$
k_{P}(h)=\frac{4}{3} h .
$$

3) $X$ is an open part of a parabola.

Remark. If we consider the distance $\delta_{P}(h)$ from the centroid of the triangle $\triangle P A B$ to the parallel line $\ell$ through the point $P+h N(P)$ to the tangent $t$ of a strictly locally convex curve $X$ at $P$, then $X$ always satisfies $\delta_{P}(h)=\frac{1}{3} h$ for all sufficiently small $h>0$.

For some characterizations of parabolas or conic sections by properties of tangent lines, see [5] and [12]. In [6], using curvature function $\kappa$ and support function $h$ of a plane curve, the first author of the present paper and Y. H. Kim gave a characterization of ellipses and hyperbolas centered at the origin.

Among the graphs of functions, Á. Bényi et al. proved some characterizations of parabolas $([1,2])$. In [15], B. Richmond and T. Richmond established a dozen necessary and sufficient conditions for the graph of a function to be a parabola by using elementary techniques.

Throughout this article, all curves are of class $C^{(3)}$ and connected, unless otherwise mentioned. 


\section{Proof of Theorem 4}

In this section, we prove Theorem 4. First of all, we need the following lemma ([9]) which is useful in the proof of main theorems.

Lemma 7. Suppose that $X$ is a strictly locally convex $C^{(3)}$ curve in the plane $\mathbb{R}^{2}$ with the unit normal $N$ pointing to the convex side. Then we have

$$
\lim _{h \rightarrow 0} \frac{1}{\sqrt{h}} L_{P}(h)=\frac{2 \sqrt{2}}{\sqrt{\kappa(P)}},
$$

where $\kappa(P)$ is the curvature of $X$ at $P$ with respect to the unit normal $N$ pointing to the convex side.

Now, we prove Theorem 4 as follows.

Let us denote by $X$ a strictly locally convex $C^{(3)}$ curve in the Euclidean plane $\mathbb{R}^{2}$. We fix an arbitrary point $P$ on $X$. Then, we may take a coordinate system $(x, y)$ of $\mathbb{R}^{2}$ such that $P$ is the origin $(0,0)$ and $x$-axis is the tangent line $t$ of $X$ at $P$. Furthermore, we may regard $X$ to be locally the graph of a non-negative strictly convex function $f: \mathbb{R} \rightarrow \mathbb{R}$ with $f(0)=f^{\prime}(0)=0$. Then $N$ is the upward unit normal.

Since the curve $X$ is of class $C^{(3)}$, the Taylor's formula of $f(x)$ is given by

$$
f(x)=a x^{2}+f_{3}(x)
$$

where $2 a=f^{\prime \prime}(0)$ and $f_{3}(x)$ is an $O\left(|x|^{3}\right)$ function. Noting that the curvature $\kappa$ of $X$ at $P$ is given by $\kappa(P)=f^{\prime \prime}(0)>0$, we see that $a$ is positive.

For a sufficiently small $h>0$, the line $\ell$ through $P+h N(P)$ and orthogonal to $N(P)$ is given by $y=h$. We denote by $A(s, f(s))$ and $B(t, f(t))$ the points where the line $\ell: y=h$ meets the curve $X$ with $s<0<t$. Then we have $f(s)=f(t)=h$. The tangent lines $t_{1}$ and $t_{2}$ to $X$ at $A$ and $B$ intersect at the point $Q=\left(x_{0}(h), y_{0}(h)\right)$ with

$$
\begin{gathered}
x_{0}(h)=\frac{t f^{\prime}(t)-s f^{\prime}(s)}{f^{\prime}(t)-f^{\prime}(s)}, \\
y_{0}(h)=h+\frac{(t-s) f^{\prime}(t) f^{\prime}(s)}{f^{\prime}(t)-f^{\prime}(s)}<0
\end{gathered}
$$

and they meet the $x$-axis (the tangent to $X$ at $P$ ) at $B_{1}\left(s-h / f^{\prime}(s), 0\right)$ and $B_{2}\left(t-h / f^{\prime}(t), 0\right)$, respectively.

Noting $L_{P}(h)=t-s$, one gets

$$
j_{P}(h)=h-\frac{1}{3}\left\{2 h+y_{0}(h)\right\}=-\frac{1}{3} \frac{L_{P}(h) f^{\prime}(t) f^{\prime}(s)}{f^{\prime}(t)-f^{\prime}(s)}
$$

and

$$
k_{P}(h)=h-\frac{1}{3} y_{0}(h)=\frac{2}{3} h-\frac{1}{3} \frac{L_{P}(h) f^{\prime}(t) f^{\prime}(s)}{f^{\prime}(t)-f^{\prime}(s)} .
$$


Hence we obtain

$$
\frac{j_{P}(h)}{h}=\frac{1}{3} \frac{L_{P}(h)}{\sqrt{h}} \frac{1}{\alpha_{P}(h)}
$$

and

$$
\frac{k_{P}(h)}{h}=\frac{2}{3}+\frac{j_{P}(h)}{h},
$$

where we use

$$
\alpha_{P}(h)=\frac{\left(f^{\prime}(s)-f^{\prime}(t)\right)}{f^{\prime}(s) f^{\prime}(t)} \sqrt{h} .
$$

On the other hand, it follows from Lemma 5 in [13] that

$$
\lim _{h \rightarrow 0} \alpha_{P}(h)=\frac{\sqrt{2}}{\sqrt{\kappa(P)}} .
$$

Together with (2.7) and Lemma 7, this shows that

$$
\lim _{h \rightarrow 0} \frac{j_{P}(h)}{h}=\frac{2}{3},
$$

and hence from (2.8) we also get

$$
\lim _{h \rightarrow 0} \frac{k_{P}(h)}{h}=\frac{4}{3} .
$$

This completes the proof of Theorem 4.

\section{Proofs of Theorems 5 and 6}

In this section, we shall prove Theorem 5 and Theorem 6 .

In order to prove Theorem 5 we use the main result of Theorem 3 in [9] and Theorem 4 stated in Section 1.

First, we prove:

Lemma 8. Suppose that $X$ is a strictly locally convex $C^{(3)}$ curve in the plane $\mathbb{R}^{2}$ with the unit normal $N$ pointing to the convex side. Then we have

$$
\sqrt{h} \frac{d}{d h} L_{P}(h)=\alpha_{P}(h),
$$

where $\alpha_{P}(h)$ is defined in (2.9).

Proof. Just as in the proof of Theorem 4 in Section 2, for an arbitrary point $P$ on $X$ we take a coordinate system $(x, y)$ of $\mathbb{R}^{2}$ so that $(2.2)$ holds with $f(0)=f^{\prime}(0)=0$ and $2 a=f^{\prime \prime}(0)>0$. Then, for sufficiently small $h>0$, we put $f(t)=h$ with $t>0$ and we denote by $A(s(t), h)$ and $B(t, h)$ the points where the line $\ell: y=h$ meets the curve $X$ with $s=s(t)<0<t$. Then we have

$$
f(s(t))=f(t)=h
$$


and

$$
L_{P}(h)=t-s(t)
$$

Noting $h=f(t)$, one obtains from (3.3) that

$$
\frac{d}{d h} L_{P}(h)=\left(1-s^{\prime}(t)\right) \frac{d t}{d h}=\frac{1-s^{\prime}(t)}{f^{\prime}(t)} .
$$

Therefore, it follows from (3.2) that

$$
\frac{d}{d h} L_{P}(h)=\frac{1}{f^{\prime}(t)}-\frac{1}{f^{\prime}(s(t))}=\frac{f^{\prime}(s)-f^{\prime}(t)}{f^{\prime}(t) f^{\prime}(s)} .
$$

Together with (2.9), this completes the proof of Lemma 8.

It is obvious that any open part of parabolas satisfy 1) and 2) in Theorem 5.

Conversely, suppose that $X$ is a strictly locally convex $C^{(3)}$ curve in the plane $\mathbb{R}^{2}$ which satisfies for all $P \in X$ and sufficiently small $h>0$

$$
j_{P}(h)=\lambda(P) h^{\mu(P)},
$$

where $\lambda(P)$ and $\mu(P)$ are some functions. Using Theorem 4 , by letting $h \rightarrow 0$ we see that

$$
\lim _{h \rightarrow 0} h^{\mu(P)-1}=\frac{2}{3} \frac{1}{\lambda(P)},
$$

which shows that $\mu(P)=1$ and $\lambda(P)=\frac{2}{3}$. Therefore, the curve $X$ satisfies for all $P \in X$ and sufficiently small $h>0$

$$
j_{P}(h)=\frac{2}{3} h .
$$

Now, using Lemma 8 we get the following.

Lemma 9. Suppose that $X$ denotes a strictly locally convex $C^{(3)}$ curve in the plane $\mathbb{R}^{2}$ which satisfies (1.5) for all $P \in X$ and sufficiently small $h>0$. Then for all $P \in X$ and sufficiently small $h>0$ we have

$$
L_{P}(h)=\frac{2 \sqrt{2}}{\sqrt{\kappa(P)}} \sqrt{h} .
$$

Proof. It follows from (1.5) and (2.7) that

$$
L_{P}(h)=2 \sqrt{h} \alpha_{P}(h) .
$$

Together with Lemma 8, this yields

$$
2 h \frac{d}{d h} L_{P}(h)=L_{P}(h) .
$$

By integrating (3.9) with respect to $h$, we get for some constant $C=C(P)$

$$
L_{P}(h)=C \sqrt{h} \text {. }
$$

Thus, Lemma 7 completes the proof of Lemma 9. 
Finally, we prove Theorem 5 as follows.

It follows from (1.2) and $S_{P}(0)=0$ that by integrating (3.7) we get

$$
S_{P}(h)=\frac{4 \sqrt{2}}{3 \sqrt{\kappa(P)}} h \sqrt{h} .
$$

Hence, together with (1.1) and (3.7), (3.11) yields that for all $P \in X$ and sufficiently small $h>0$

$$
S_{P}(h)=\frac{4}{3} T_{P}(h)
$$

Thus, it follows from Proposition 2 that $X$ is an open part of a parabola. This completes the proof of Theorem 5 .

In order to prove Theorem 6 , with the help of (2.8) we may use the similar argument as in the proof of Theorem 5 .

\section{References}

[1] Á. Bényi, P. Szeptycki, and F. Van Vleck, Archimedean properties of parabolas, Amer. Math. Monthly 107 (2000), no. 10, 945-949.

[2] _ A generalized Archimedean property, Real Anal. Exchange 29 (2003/04), no. 2, 881-889.

[3] W. A. Day, Inequalities for areas associated with conics, Amer. Math. Monthly 98 (1991), no. 1, 36-39.

[4] M. P. do Carmo, Differential Geometry of Curves and Surfaces, Prentice-Hall, Englewood Cliffs, NJ, 1976.

[5] D.-S. Kim and S. H. Kang, A characterization of conic sections, Honam Math. J. 33 (2011), no. 3, 335-340.

[6] D.-S. Kim and Y. H. Kim, A characterization of ellipses, Amer. Math. Monthly 114 (2007), no. $1,66-70$.

[7] _ Some characterizations of spheres and elliptic paraboloids, Linear Algebra Appl. 437 (2012), no. 1, 113-120.

[8] _ Some characterizations of spheres and elliptic paraboloids II, Linear Algebra Appl. 438 (2013), no. 3, 1356-1364.

9] _ On the Archimedean characterization of parabolas, Bull. Korean Math. Soc. 50 (2013), no. 6, 2103-2114.

[10] D.-S. Kim, W. Kim, Y. H. Kim, and D. H. Park, Area of triangles associated with a curve II, Bull. Korean Math. Soc. 52 (2015), no. 1, 275-286.

[11] D.-S. Kim, Y. H. Kim, and S. Park, Center of gravity and a characterization of parabolas, Kyungpook Math. J., To appear; arXiv:1502.00188.

[12] D.-S. Kim, J. H. Park, and Y. H. Kim, Some characterizations of parabolas, Kyungpook Math. J. 53 (2013), no. 1, 99-104.

[13] D.-S. Kim and K.-C. Shim, Area of triangles associated with a curve, Bull. Korean Math. Soc. 51 (2014), no. 3, 901-909.

[14] J. Krawczyk, On areas associated with a curve, Zesz. Nauk. Uniw. Opol. Mat. 29 (1995), 97-101.

[15] B. Richmond and T. Richmond, How to recognize a parabola, Amer. Math. Monthly 116 (2009), no. 10, 910-922.

[16] S. Stein, Archimedes. What did he do besides cry Eureka?, Mathematical Association of America, Washington, DC, 1999. 
DONG-SOO KIM

Department of Mathematics

Chonnam National University

GWANGJU 500-757, Korea

E-mail address: dosokim@chonnam.ac.kr

Dong Seo Kim

Department of Mathematics

Chonnam National University

Gwanguu 500-757, Korea

E-mail address: dongseo@chonnam.ac.kr 